09

\title{
Исследование концентраторных фотоэлектрических модулей с каскадными солнечными элементами
}

\author{
() Е.А. Ионова, Н.Ю. Давидюк, Н.А. Садчиков, А.В. Андреева \\ Физико-технический институт им. А.Ф. Иофрфе РАН, \\ 194021 Санкт-Петербург, Россия \\ e-mail: ionova@mail.ioffe.ru
}

Поступило в Редакцию 31 марта 2021 г.

В окончательной редакции 20 апреля 2021 г.

Принято к публикации 21 апреля 2021 г.

При исследовании концентраторных фотоэлектрических модулей с трехпереходными солнечными элементами на основе структуры GaInP/GaInAs/Ge использованы измерительные возможности имитатора солнечного излучения и компьютерное моделирование. Показана возможность совместного использования двух методов для объяснения процессов, происходящих при преобразовании излучения в системе концентратор-солнечный элемент, и прогнозирования параметров модуля при изменении внешних условий фокусировки. Установлен вклад латеральных токов в формирование фототока солнечного элемента в модуле. Для системы линза Френеля-каскадный солнечный элемент определены возможные причины энергетических потерь за счет внутреннего сопротивления в солнечных элементах. Методом компьютерного моделирования рассчитаны выходные характеристики модуля при различных спектрах падающего излучения.

Ключевые слова: линза Френеля, солнечный элемент, кристаллический кремний.

DOI: $10.21883 / J T F .2021 .09 .51223 .89-21$

\section{Введение}

Большая часть солнечных батарей в мире производится с использованием стандартной технологии кристаллического кремния. Эффективность кремниевых солнечных батарей составляет 20\% при незначительном потенциале роста [1]. В сложившихся условиях конкурировать на рынке солнечных батарей может исключительно высокотехнологичная продукция нового поколения, превосходящая по своим характеристикам традиционные кремниевые батареи. Одним из наиболее перспективных направлений солнечной фотоэнергетики является преобразование солнечного излучения с использованием высокоэффективных каскадных солнечных элементов (СЭ) и недорогих оптических концентраторов [2-4]. Применение концентраторов позволяет снижать расход полупроводникового материала пропорционально степени концентрирования солнечного излучения. Использование концентраторных фотоэлектрических модулей (КФЭМ) с установками слежения за Солнцем делает возможным увеличение суточного энергосъема с единицы площади солнечной батареи по сравнению с традиционными кремниевыми батареями.

Ряд мировых компаний, занимающихся разработкой многокаскадных солнечных элементов, достигли значительных результатов в увеличении эффективности преобразования солнечного излучения. Максимальное значение эффективности преобразования солнечного излучения в 6-каскадных СЭ достигает в настоящее время $47.1 \%$ в условиях равномерной освещенности поверхно- сти СЭ излучением с эталонным спектром AM1.5D и интенсивностью, соответствующей 143-кратному уровню концентрирования [5]. Значения КПД трехкаскадных солнечных элементов, производимых в настоящее время в промышленных объемах, составляет 42-43\% [6].

На сегодняшний день при разработке КФЭМ наиболее широкое распространение получило использование линз Френеля малой, менее $1 \mathrm{~mm}$, толщины в качестве первичных концентраторов солнечного излучения. Концентраторные модули с линзами Френеля обладают рядом конструктивных преимуществ, связанных с планарностью концентраторной панели, упрощающей конструкцию КФЭМ, и обеспечивающей защиту внутреннего объема модулей от воздействий внешней среды $[4,7,8]$.

Лучшие значения КПД промышленных образцов концентраторных модулей достигают 32\% при 400-700кратной степени концентрирования излучения $[9,10]$.

В настоящей работе были проведены исследования характеристик КФЭМ с концентраторной системой на основе линзы Френеля и трехкаскадного солнечного элемента с целью разработки КФЭМ с КПД, максимально приближенным к КПД СЭ.

\section{1. Задача исследования}

Эффективность преобразования солнечного излучения КФЭМ в значительной степени зависит от характеристик концентраторной системы. В нашей предыдущей работе [11] было показано, что для расчета эффек- 
тивности фокусировки излучения в концентраторных модулях на основе каскадных СЭ необходимо исследовать характеристики работы концентратора в паре с соответствующим СЭ.

В настоящей работе рассматривается работа концентраторного фотоэлектрического модуля с одной парой „концентратор-солнечный элемент ${ }^{6}$. Для преобразования излучения был использован трехкаскадный солнечный элемент на основе структуры GaInP/GaInAs/Ge, содержащий в одном кристалле три соединенных последовательно субэлемента со своими $p-n$-переходами [6].

Моделирование и конструирование КФЭМ с наибольшим КПД преобразования солнечного излучения сопряжено с решением задачи достижения наибольших значений фактора заполнения (FF) световой вольт-амперной характеристики (BAX) солнечного элемента в модуле при сохранении наибольшего тока короткого замыкания (ТК3), $I_{s c}$. При этом условии мощность вблизи точки оптимальной нагрузки изменяется пропорционально изменению FF.

В используемом каскадном СЭ с тремя субэлементами генерация фототока происходит аналогично последовательно соединенным трем источникам тока, т.е. фототок СЭ определяется наименьшим (ограничивающим) фототоком, генерируемым в $p-n$-переходах одного из трех субэлементов. Распределение плотности фототока по фотоприемной поверхности СЭ в каждом субэлементе определяется распределением излучения с длинами волн, относящихся к диапазону поглощения соответствующего субэлемента, которое, в свою очередь, зависит от свойств концентратора. Световое пятно в фокусе концентратора, из-за дисперсии показателя преломления материала концентратора, в трех спектральных диапазонах, относящихся к трем субэлементам, имеет различный размер и картину возрастания-убывания концентрации фотонов, эффективно поглощающихся в субэлементах. В настоящей работе рассматривается влияние несовпадения в субэлементах распределений концентрации фотонов, генерирующих носители тока, на выходные характеристики модуля.

Известно, что по мере изменения высоты Солнца над горизонтом меняется толщина атмосферной массы (АМ), сквозь которую проходит солнечное излучение. При изменении спектра Солнца от AM1 до AM6 (зенит-закат) плотность мощности излучения в спектральных диапазонах, относящихся к поглощению в первом, втором и третьем субэлементах уменьшается соответственно в 3, 1.7, 1.5 раза. Преимущественно уменьшается доля солнечной энергии, преобразуемой первым субэлементом. По мере роста АМ может произойти смена субэлемента, ограничивающего фототок СЭ. В настоящей работе вычисляются и анализируются значения ТКЗ, генерируемые субэлементами при разных спектрах падающего излучения. Массивы данных, определяющих спектры солнечного излучения, получены с помощью программы SMARTS.

\section{2. Реальный модуль и модель для расчета характеристик}

Исследуемый модуль содержит линзу Френеля размерами $60 \times 60 \mathrm{~mm}$ и СЭ размерами $3.1 \times 3.5 \mathrm{~mm}$ (фотоприемная область $3 \times 3 \mathrm{~mm}$ ) фирмы Azur с антиотражающим покрытием, оптимизированным под границу перехода излучения СЭ-воздух. Для изготовления линзы Френеля на прецизионном станке методом алмазного точения была изготовлена металлическая матрица, с помощью которой была сформирована реплика линзы из прозрачной силиконовой резины Elastosil RT604 фирмы Wacker на стеклянной пластине повышенной прозрачности толщиной $4 \mathrm{~mm}$. Профиль линзы Френеля, представляющий собой набор кольцевых конических преломляющих поверхностей, был рассчитан под фокусное расстояние $F$, равное $105 \mathrm{~mm}$, выбранное из условия попадания на СЭ всего фокусного пятна.

Функционирование модели модуля рассчитывается с помощью вычислительной программы (ВП), составленной методом процедурного программирования на языке $\mathrm{C}++$.

Параметры модели соответствуют параметрам реального модуля, но модель представляет идеализированный вариант реального модуля, так как среди всех возможных микродефектов модуля в модели учитываются только оптическое поглощение в концентраторе и два вида технологически обусловленных дефектов профиля концентратора. ВП совершает множество однотипных операций по моделированию хода лучей через модель модуля. Принимаются в расчет все значимые характеристики падающего излучения, концентратора и СЭ, в числе которых угловая расходимость и спектральная плотность мощности падающего излучения, размер, шаг профиля линзы Френеля, расстояние между линзой и СЭ, углы наклона преломляющих граней, дисперсия показателя преломления силиконовой резины и зависимость дисперсии от температуры, отражение от концентратора, спектральная характеристика внешнего квантового выхода СЭ.

\section{3. Метод исследования}

Исследования КФЭМ проводятся методами компьютерного моделирования (КМ) и прямых измерений, а также синтеза результатов применения этих методов, который возможен при установлении совпадения результатов, полученных методами КМ и прямых измерений.

Проверка совпадения необходима, так как в случае значительных неучтенных дефектов концентратора, ошибок в измерениях или вычислительной программе, синтез результатов исследований будет ошибочным.

Прямые измерения выходных характеристик реального модуля проводились с помощью имитатора солнечного излучения (ИСИ), в котором излучение импульсной ксеноновой лампы-вспышки преобразуется в световой 


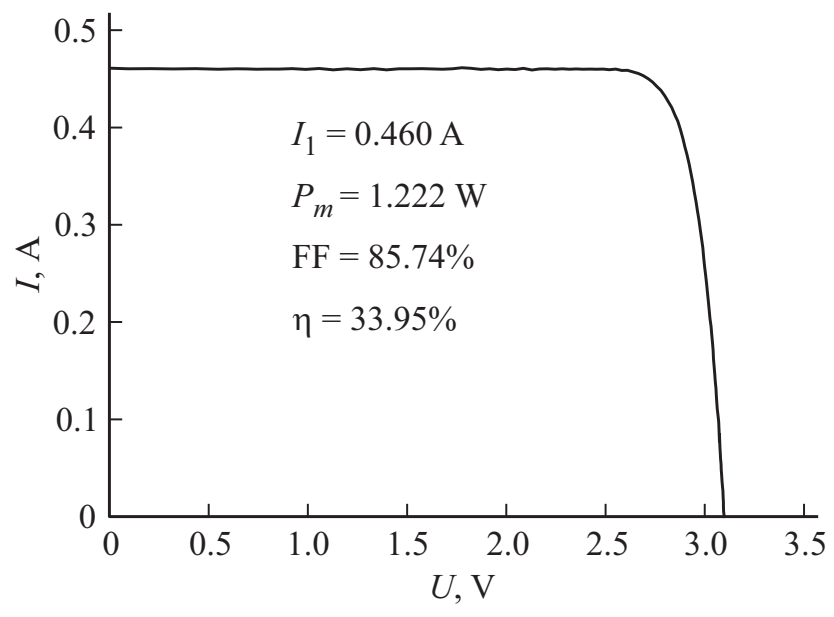

Pис. 1. BAX пары концентратор-СЭ, измеренная на имитаторе солнечного излучения. $I_{1}-$ ток короткого замыкания, $P_{m}$ - мощность в точке оптимальной нагрузки, FF - фактор заполнения ВАХ, $v$ - КПД пары концентратор-СЭ.

пучок со спектральным составом и угловой расходимостью, соответствующими расходимости и спектру солнечного излучения AM1.5D с плотностью излучения $1 \mathrm{~kW} / \mathrm{m}^{2}$. ИСИ содержит систему крепления, обеспечивающую ориентацию элементов модуля в световом потоке, их взаимное перемещение по трем координатам с прецизионной точностью и измерительный блок. При проведении измерений линза Френеля закреплялась на держателе имитатора солнечного излучения, плоскость линзы Френеля устанавливалась перпендикулярно направлению падающего излучения. На расстоянии $105 \mathrm{~mm}$ закреплялся СЭ, так, что его центр был расположен на оптической оси линзы Френеля.

На рис. 1 приведена ВАХ исследуемой пары концентратор-СЭ, записанная при облучении световым потоком от ксеноновой лампы-вспышки. ТКЗ модуля $I_{1}$ составил 0.460 А. Расчет с помощью ВП числа сгенерированных носителей тока в трех субэлементах показал, что ТКЗ модели модуля I2 равен 0.465 А. То есть расчетный и измеренные фототоки СЭ практически совпадают. Имеющая место разница между значениями в $1 \%$ представляет собой накопленную погрешность измерений ИСИ и данных для ВП.

Для качественной оценки полученных результатов были проведены дополнительные элементарные вычисления без использования ВП: спектр ксеноновой лампывспышки был умножен на спектр поглощения концентратора и на спектральную характеристику внешнего квантового выхода, проинтегрирован и нормирован на площадь концентратора $60 \times 60 \mathrm{~mm}$. Полученное в результате значение тока $0.554 \mathrm{~A}$ было умножено на коэффициент 0.92, определяющий отражение от концентратора, скорректировано коэффициентом потерь оптического излучения $(<3 \%)$ на известных технологически обусловленных дефектах профиля концентратора и коэффициентом затенения контактной сеткой СЭ (6\%). В результате расчетов было получено оценочное максимальное значение ТКЗ у исследуемого СЭ при преобразовании излучения, концентрированного линзой с апертурой $60 \times 60 \mathrm{~mm}$, которое составляло $0.464 \mathrm{~A}$. Таким образом, можно заключить, что расчетный и измеренные фототоки СЭ не только совпадают между собой, но и совпадают с максимально возможным ТКЗ в модуле с данным концентратором и СЭ.

С помощью ИСИ было определено распределение кратности увеличения фототока (КУФ) в фокусном пятне на фотоприемной поверхности СЭ вдоль линии, включающей максимум фототока. Для этого на СЭ устанавливалась диафрагма с отверстием, открывающим на СЭ округлую область без контактной сетки площадью $0.0085 \mathrm{~mm}^{2}$. При построении распределения КУФ, СЭ с установленной диафрагмой дискретно перемещался вдоль линии, проходящей через центр сфокусированного пятна, после каждого перемещения производилась вспышка ксеноновой лампы, и записывалось значение ТКЗ. Кратность увеличения фототока определялась как результат деления плотности ТКЗ СЭ, измеренного при засветке концентрированным излучением через отверстие диафрагмы, на плотность ТКЗ, измеренного при вспышке без концентрирования излучения на СЭ, без диафрагмы (рис. 2, кривая 1).

Распределение КУФ в модели модуля вычисляется программой отдельно для трех субэлементов. На рис. 3 показано распределение КУФ вдоль линии, проходящей через центр фокального пятна (кривые 1-3). Общее для всего СЭ распределение КУФ в фокусном пятне строилось в приближении отсутствия токов вдоль полупроводниковых слоев и определялось по распределениям КУФ для субэлементов выбором участка кривой, наименьшего по значениям КУФ, и затем корректировалось с учетом потерь оптического излучения (рис. 3,

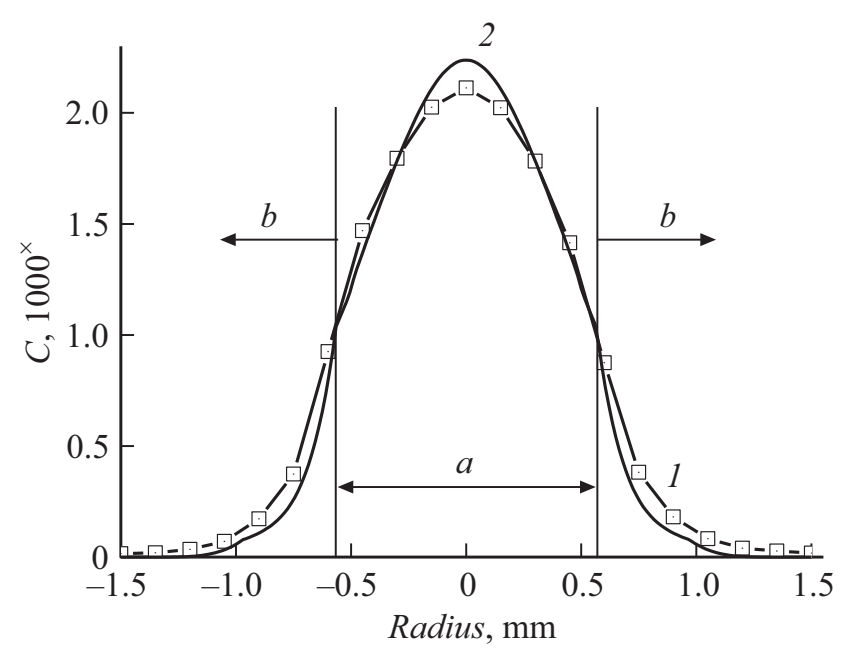

Рис. 2. Измеренная кратность увеличения фототока в фокусном пятне концентратора (кривая 1) и вычисленное распределение кратности увеличения фототока (кривая 2). 


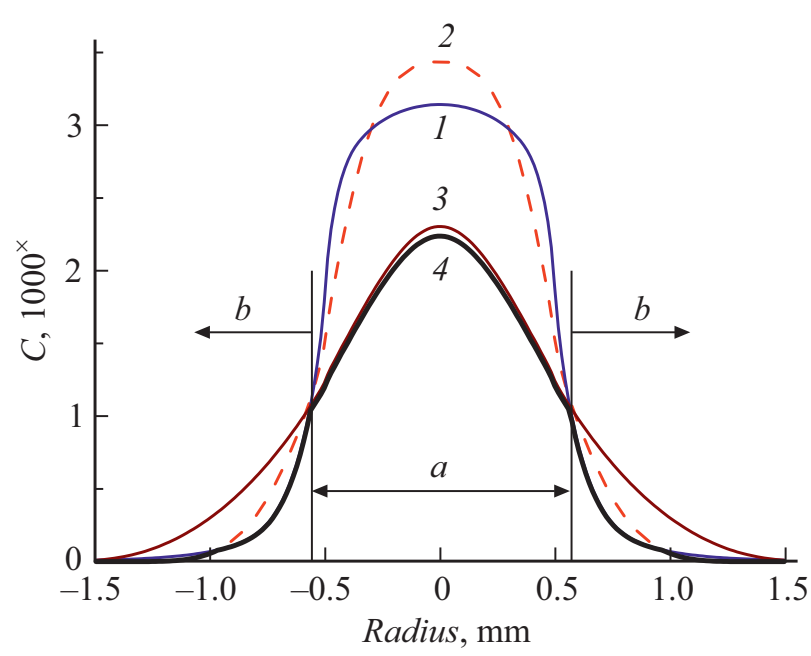

Рис. 3. Вычисленные кривые распределения кратности увеличения фототока в фокусе концентратора вдоль линии середины СЭ для первого GaInP, второго GaInAs, третьего Ge субэлементов (кривые 1-3) и общее для СЭ распределение кратности увеличения фототока с учетом оптических потерь исследуемого концентратора (кривая 4).

кривая 4). Данная кривая распределения КУФ в модели модуля для удобства сравнения перенесена на график распределения КУФ в фокусном пятне реального модуля (рис. 2). Следует отметить, что в распределение КУФ в реальном модуле, измеренном с помощью ИСИ (рис. 2, кривая 1), также не вносят вклад токи вдоль полупроводниковых слоев, так как генерация носителей во всех трех субэлементах модуля происходит в областях очень малой площади, расположенных соосно с отверстием диафрагмы, остальная часть СЭ остается затененной.

На рис. 2 видно, что размер фокусного пятна, области возрастания и убывания распределения КУФ, его максимум практически совпадают.

Равенство ТКЗ СЭ и совпадение распределений КУФ, измеренных на ИСИ в реальном модуле и вычисленных для модели модуля в идеализированном варианте, позволяет сделать вывод о достоверности результатов теоретических расчетов параметров модели модуля. Высокая степень совпадения кривых распределений КУФ, полученных экспериментально и путем вычислений, свидетельствует об отсутствии в исследуемом концентраторе значительных неучтенных дефектов, которые могли бы привести к размытию фокусного пятна.

\section{4. Латеральные токи}

В качестве первого примера совместного использования результатов теоретического и практического исследований было рассмотрено влияние спектрального расслоения фокусного пятна на работу каскадного СЭ, возникающее из-за дисперсии показателя преломления материала концентратора [12]. Как видно из соотноше- ний распределений КУФ на рис. 3, вычисленных для модели модуля, фототок в направлении к выводным контактам СЭ, генерируемый в центральной зоне $(a)$ диаметром $1.12 \mathrm{~mm}$, не должен быть больше фототока третьего субэлемента, а фототок в направлении к выводным контактам СЭ в окружающей области (b) ограничен фототоком первого субэлемента. Если бы сгенерированные носители тока в центральной области в первом и втором субэлементах, а на периферии во втором и третьем субэлементах - не вносили вклад в общий фототок СЭ, то, как это легко вычислить с помощью ВП, общий фототок модели модуля $I_{2}^{\prime}$ должен был составлять $0.344 \mathrm{~A}$. Это значение ТКЗ намного меньше полученного экспериментальным и вычислительным методами.

Произведенные расчеты свидетельствуют о 26\% вкладе в ТКЗ модуля латеральных токов, т. е. противоположно направленного движения сгенерированных носителей из центральной области (a) (рис. 3) на периферию, а из крайних областей $(b)-$ по направлению к центру. При этом можно заключить, что вклад латеральных токов в указанном размере не влияет на величину ТКЗ модуля, который определяется суммарной концентрацией сгенерированных носителей в субэлементе, дающем наименьший фототок.

В центральной области фотоприемной поверхности СЭ площадью $\sim 1 \mathrm{~mm}^{2}$ имеет место разница порядка $1000^{\times}$между КУФ, вычисленных для первого и второго субэлементов, и КУФ, вычисленной для третьего субэлемента, а в крайних областях СЭ площадью $\sim 8 \mathrm{~mm}^{2}$ разница между КУФ для третьего субэлемента и двух других не больше $300^{\times}$(рис. 3). Распределение плотности фотогенерированных носителей тока в субэлементах таково, что преобладающим должно быть радиальное движение латеральных токов в направлении от центра.

Таким образом, в КФЭМ с каскадными СЭ и концентраторами, дающими спектральное расслоение фокусного пятна, важную роль играет как центральная область СЭ, дающая наибольший фототок к выводным контактам по причине большей плотности энергии излучения в центре фокусного пятна, так и периферийные области СЭ, фототок от которых включает латеральные токи, компенсирующие сниженный вклад в общую генерацию центральных зон первого и второго субэлементов.

\section{5. Фактор заполнения ВАХ}

В качестве второго примера использования результатов теоретического и практического исследований была рассмотрена связь между рассчитанным спектральным расслоением фокусного пятна и измеренным фактором заполнения BAX солнечного элемента (FF). При исследованиях на ИСИ было замечено, что при варьировании расстояния $F^{\prime}$ между концентратором и СЭ значительно меняется FF. Причина этого состоит в росте потерь на внутренних сопротивлениях каскадного СЭ, который 


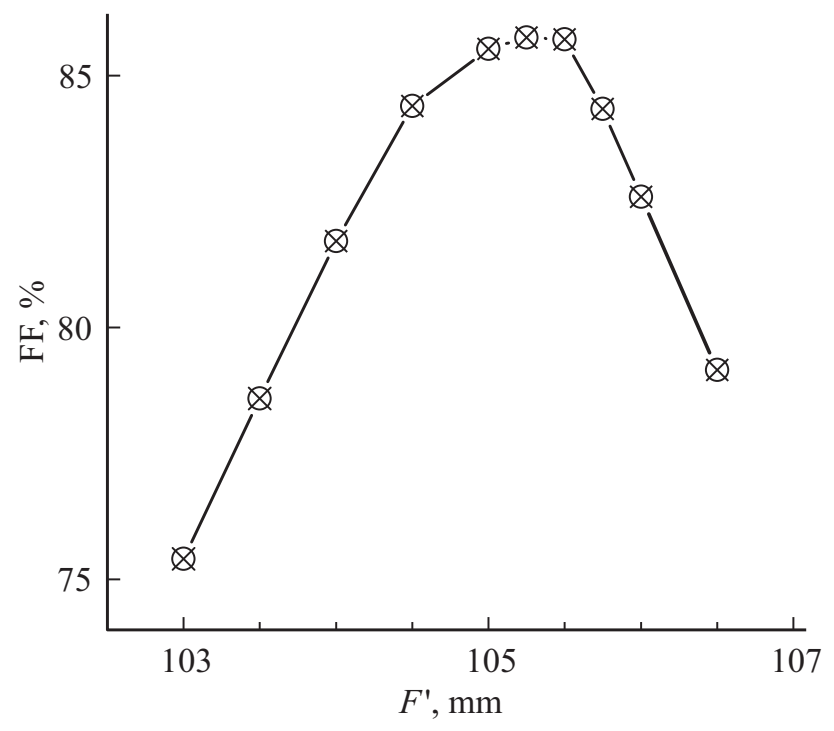

Рис. 4. Фактор заполнения ВАХ в зависимости от расстояния между концентратором и СЭ.

происходит одновременно со спектральным перераспределением плотности излучения на фотоприемной поверхности при варьировании расстояния до СЭ.

На рис. 4 приведены результаты измерений выходных характеристик пары концентратор-СЭ, проведенных с помощью ИСИ при разных расстояниях между концентратором и СЭ. Фактор заполнения ВАХ имеет выраженный максимум в интервале $105-105.5 \mathrm{~mm}$, генерируемая мощность в точке оптимальной нагрузки максимальна в интервале $104.75-105.5 \mathrm{~mm}$. Она снижается примерно на 5\% от максимума при отклонении расстояния в паре концентратор-СЭ от фокусного на $\pm 1 \mathrm{~mm}$. ТКЗ СЭ при этом оставался постоянным, т.е. излучение из участка спектра, относящегося к субэлементу, ограничивающему ТКЗ, за пределы СЭ не выходит.

При теоретическом исследовании процесса для модели модуля были вычислены распределения плотности ТКЗ $J$ по фотоприемной поверхности СЭ при варьировании расстояния между концентратором и СЭ, как параметра. Рассчитанные величины ТКЗ, так же как и при измерениях, остаются постоянными. На графиках, приведенных на рис. 5, можно наблюдать, что при варьировании $F^{\prime}$ в интервале 104-106 mm, вследствие изменения в спектральной структуре светового пятна на фотоприемной поверхности СЭ, формы распределений плотности ТКЗ, построенных для трех субэлементов, значительно меняются.

Сопоставляя результаты компьютерного моделирования и измерений на ИСИ, можно установить, что:

во-первых, максимальный FF при $F^{\prime}=105.25 \mathrm{~mm}$ соответствует практически сравнявшимся распределениям плотности ТКЗ для первого и второго субэлементов при сравнительно небольшом значении максимума плотности ТКЗ для третьего субэлемента $-27 \mathrm{~A} / \mathrm{cm}^{2}$. При таком распределении плотностей ТКЗ латеральные токи в слоях между первым и вторым субэлементами отсутствуют. Латеральные токи, протекающие в слоях между вторым и третьим субэлементами, не приводят к возникновению заметных дополнительных потерь при протекании тока вследствие относительно большой толщины слоев $\mathrm{Ge}$ субэлемента и их малого сопротивления [13];

во-вторых, при отклонении расстояния $F^{\prime}$ от фокусного, убыванию FF сопутствует увеличение разницы

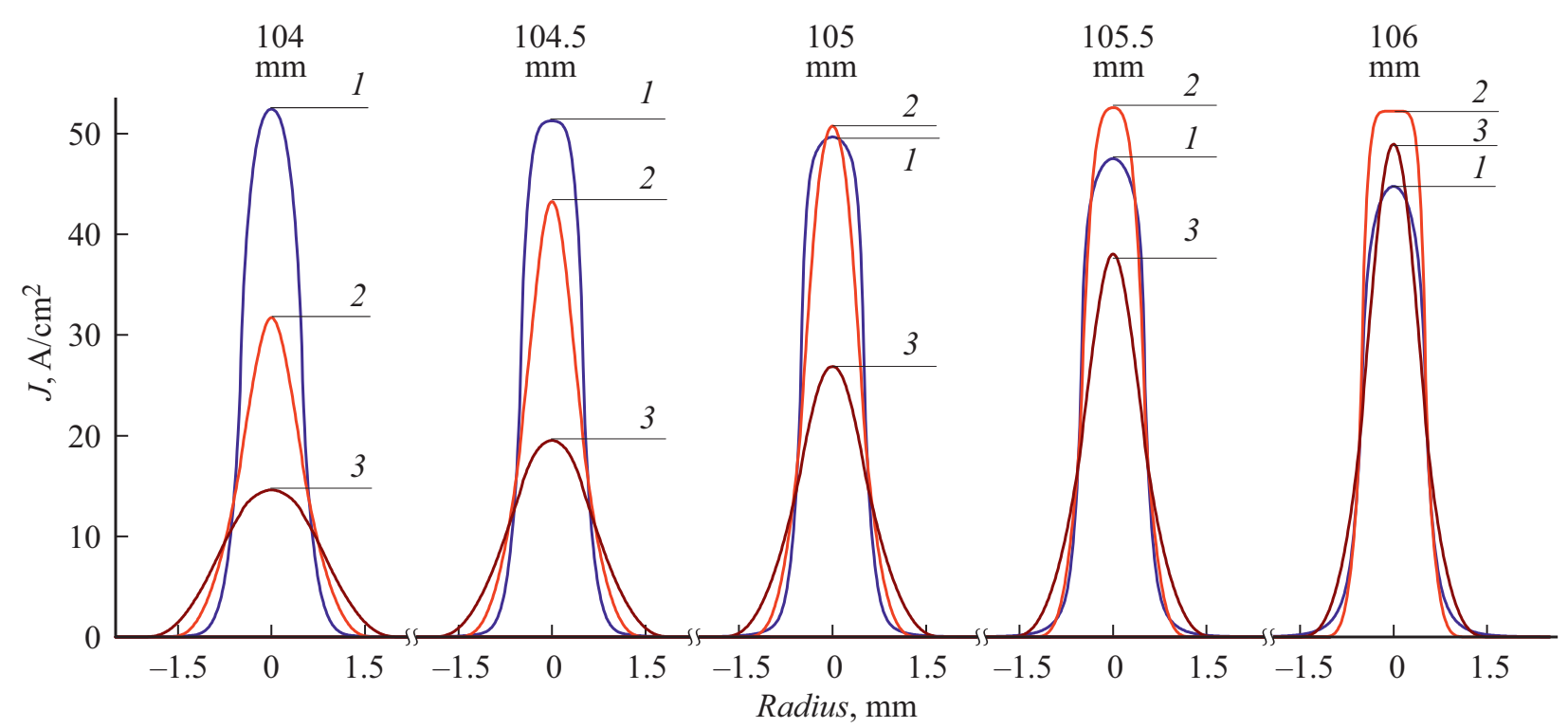

Рис. 5. Вычисленное распределение плотности тока короткого замыкания $J$ вдоль линии середины СЭ в трех субэлементах модели при разных расстояниях $F^{\prime}$ между концентратором и СЭ при фокусировке излучения со спектром ксеноновой лампы-вспышки. Кривая 1 - первый GaInP субэлемент, кривая 2 - второй GaInAs субэлемент, кривая 3 - третий Ge субэлемент. 


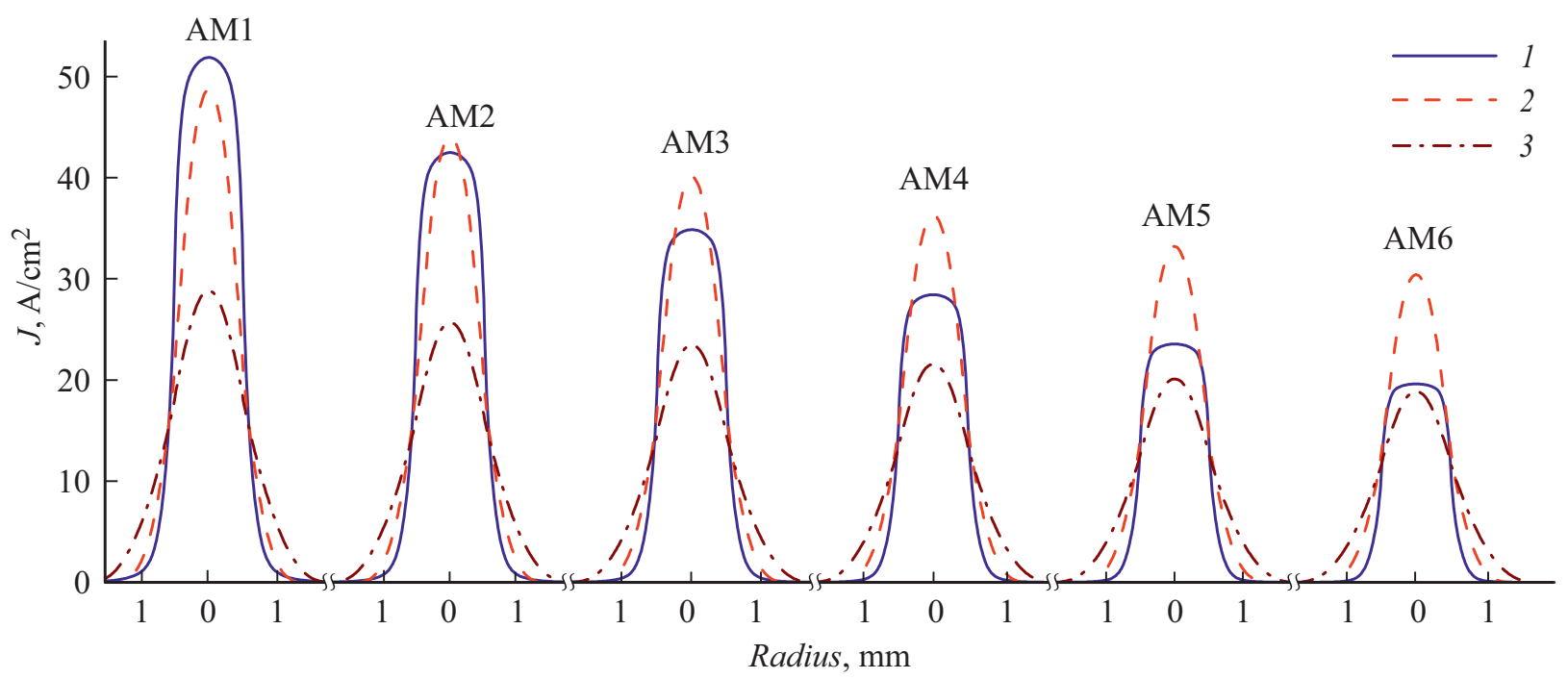

Рис. 6. Вычисленное распределение плотности тока короткого замыкания в трех субэлементах модели модуля в условиях фокусировки солнечного излучения со спектрами AM1-AM6 (кривая 1 - первый GaInP субэлемент, кривая 2 - второй GaInAs субэлемент, кривая 3 - третий Ge субэлемент).

между распределениями плотности ТКЗ для первого и второго субэлемента. В этом случае возникновение латеральных токов в сравнительно тонких пограничных слоях первого и второго субэлементов может приводить к увеличению потерь на сопротивлениях этих слоев при протекании токов к контактам;

в-третьих, если расстояние $F^{\prime}$ увеличивается относительно фокусного, убыванию FF сопутствует значительное возрастание ограничивающего фототока $\mathrm{Ge}$ субэлемента в центральной области диаметром $0.5 \mathrm{~mm}$ до $49 \mathrm{~A} / \mathrm{cm}^{2}$ при $F^{\prime}=106 \mathrm{~mm}$, что соответствует возрастанию КУФ до $3000^{\times}$. Значительное возрастание плотности фототока в центре фотоприемной поверхности СЭ может приводить к возникновению дополнительных потерь на сопротивлениях контактной сетки при протекании фототока к токосборной шине;

и, наконец, в-четвертых, если расстояние $F^{\prime}$ уменьшается относительно фокусного, убыванию FF сопутствует значительное уширение фокусного пятна, относящегося к третьему субэлементу. Это приводит к тому, что латеральные токи распространяются на большие расстояния, давая дополнительные потери на сопротивлениях.

Таким образом, при моделировании КФЭМ с каскадными СЭ для достижения наибольшей генерации вблизи точки оптимальной нагрузки следует учесть, что разница между распределениями плотности ТКЗ в первом и втором субэлементах должна быть минимальной, при этом разница между ними и распределением плотности ТКЗ в третьем субэлементе имеет меньшее значение.

При разработке технологии изготовлении КФЭМ нужно учитывать, что изменение расстояния между концентратором и СЭ относительного фокусного больше, чем на $1 \mathrm{~mm}$, приводит к более чем 4\% снижению значений фототока вблизи точки оптимальной нагрузки, обуслов- ленного как уменьшением FF, так и уменьшением ТКЗ из-за того, что часть излучения начинает выходить за пределы фотоприемной поверхности.

\section{6. Исследование модели концентратор-СЭ при изменении параметров падающего излучения}

Тестирование работы фотоэлектрического модуля в естественной среде в условиях изменяющейся атмосферной массы, как параметра солнечного излучения, представляет сложность из-за меняющейся облачности и температуры. Совпадение результатов теоретических исследований работы модели модуля с результатами измерений позволяет использовать метод компьютерного моделирования для определения зависимости выходных параметров разрабатываемого КФЭМ от параметров преобразуемого солнечного излучения.

Солнечное излучение непрерывно меняет свой спектр в течение светового дня из-за изменения длины пути излучения сквозь атмосферу. Изменение спектра преломляемого излучения приводит к изменению спектрального состава фокусного пятна, а значит и соотношения распределений в плоскости СЭ плотности ТКЗ субэлементов. Для группы спектров с АМ, равной 1, 2, $3,4,5,6$, для модели модуля были вычислены распределения плотностей ТКЗ в трех субэлементах СЭ. Как показано на графиках рис. 6, по мере увеличения АМ существенно увеличивается разница между формами распределений плотности ТКЗ для первого и второго субэлементов. Но при АМ, большей 2, и в центральной и в крайних областях СЭ значения плотности ТКЗ для второго субэлемента практически на всей фотоприемной поверхности СЭ превышает значения плотности ТКЗ 


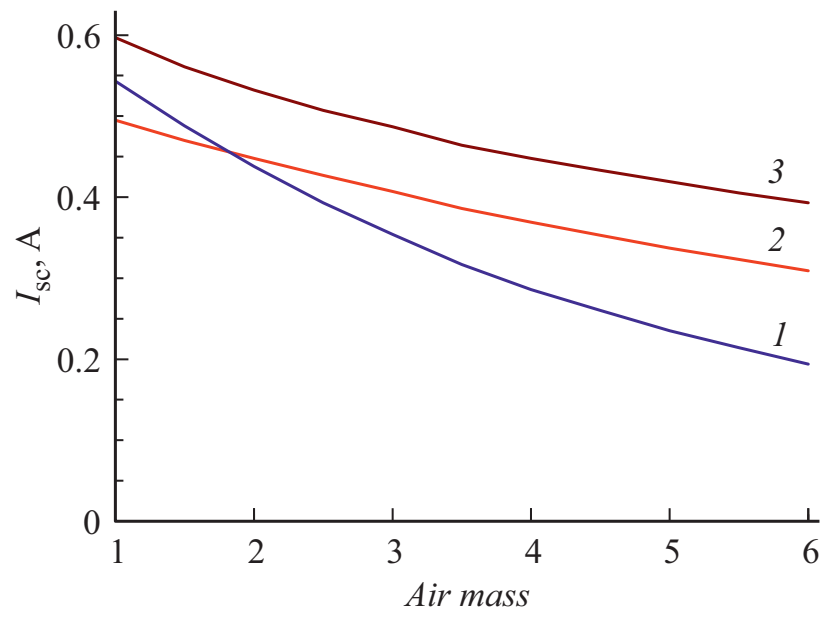

Рис. 7. Зависимости значений тока короткого замыкания в трех субэлементах модели концентратор-СЭ от спектрального состава падающего излучения (линии 1 - первый GaInP субэлемент, 2 - второй GaInAs субэлемент, 3 - третий Ge субэлемент).

для первого субэлемента. Это значит, что при росте АМ перераспределением сгенерированных носителей за счет латеральных токов в первом и втором субэлементах можно пренебречь. Исходя из предположения, что на рост потерь на внутренних сопротивлениях СЭ оказывает наибольшее влияние увеличение латеральных токов между слоями в первом и втором субэлементе, можно заключить, что потери на внутренних сопротивлениях в каскадном СЭ не увеличиваются при изменении спектра солнечного излучения при возрастании АМ.

По мере изменения спектра солнечного излучения с ростом АМ преимущественно снижается мощность излучения из участка спектра, относящегося к первому субэлементу. Для того чтобы в этих условиях оценить снижение ТКЗ СЭ, для модели модуля были произведены вычисления ТКЗ трех субэлементов при изменении $\mathrm{AM}$ от 1 до 6 с шагом 0.5 (рис. 7). По результатам вычислений при преобразовании солнечного излучения со спектрами в интервале от AM1 до AM1.8, ток короткого замыкания СЭ ограничивает ТКЗ второго субэлемента, он снижается при изменении спектра на $10 \%$ от максимума. При изменении спектра от АМ1.8 до АМ6 фототок СЭ ограничивает ТКЗ первого субэлемента, и он снижается еще на $50 \%$.

Причиной снижения фототока КФЭМ с каскадными СЭ в периоды рассвета и заката является снижение мощности потока солнечного излучения, а не свойства фокусирующей системы, так как параметры концентратора и СЭ подбирались так, чтобы сфокусированное излучение спектра AM1.5D полностью попадало на СЭ. Фокусное пятно даже уменьшается при уменьшении вклада излучения, преобразуемого первым субэлементом, так как коротковолновое излучение преломляется с показателями преломления, расположенными на крутой ветви дисперсионной зависимости, т.е. намного более высокими, чем показатели преломления излучения основной части спектра, находящиеся на пологой ветви дисперсионной зависимости материала концентратора.

\section{Заключение}

Проведены исследования характеристик модуля, состоящего из трехкаскадного солнечного элемента размером $3 \times 3 \mathrm{~mm}$ и линзы Френеля размером $60 \times 60 \mathrm{~mm}$ с фокусным расстоянием $105 \mathrm{~mm}$, с помощью имитатора солнечного излучения. Методом компьютерного моделирования проведены вычисления характеристик модели модуля с параметрами, соответствующими реальному модулю.

Сопоставление полученных результатов позволяет сделать вывод о соответствии результатов, получаемых методами компьютерного моделирования работы модели модуля, выходным характеристикам реального модуля и о возможности использования данного метода для объяснения процессов, происходящих при фокусировке излучения, а также о возможности предварительных расчетов характеристик реального модуля при разных внешних условиях при преобразовании солнечного излучения.

Исследование пары линза Френеля-каскадный солнечный элемент расчетным и экспериментальным методами позволило установить возможные причины дополнительных потерь на внутренних сопротивлениях СЭ за счет латеральных токов. Дополнительные потери на внутренних сопротивлениях рассматриваемого СЭ могут быть связаны, во-первых, с координатным несовпадением между распределениями плотности токов короткого замыкания преимущественно в первом и втором субэлементах СЭ и, во-вторых, с возрастанием максимума распределения плотности фототоков в центре фотоприемной поверхности СЭ.

Проведено исследование работы модели модуля при разных спектрах падающего излучения. Показано, что при увеличении атмосферной массы, как параметра падающего излучения, потери на внутренних сопротивлениях СЭ не увеличиваются. Установлено, что фототок солнечного элемента ограничивается фототоком второго субэлемента в интервале АM1-AM1.8, при большей АМ ограничивающим является фототок первого субэлемента.

В концентраторном фотоэлектрическом модуле, исследованном в работе, было получено значение КПД преобразования излучения $33.95 \%$, что соответствует уровню лучших мировых достижений.

\section{Конфликт интересов}

Авторы заявляют, что у них нет конфликта интересов. 


\section{Список литературы}

[1] [link] Photovoltaics-Report. Last accessed: 20.02.2021. https:/www.ise.fraunhofer.de/content/dam/ise/de/documents/ publications/studies/Photovoltaics-Report.pdf

[2] V.M. Andreev, V.A. Grilikhes, V.D. Rumyantsev. Photovoltaic Conversion of Concentrated Sunlight (J. Wiley \& Sons Ltd, NY., 1997)

[3] Zh.I. Alferov, V.M. Andreev, V.D. Rumyantsev. III-V Heterostructures in Photovoltaics. In: Concentrator Photovoltaics, eds. A. Luque, V. Andreev, (Springer Series in Optical Sciences, 2007), v. 130, p. 25.

[4] V.D. Rumyantsev, V.M. Andreev, A.W. Bett, F. Dimroth, M. Hein, G. Lange, M.Z. Shvarts, O.V. Sulima. In: Proc. of the 28th IEEE PVSC, Alaska, 1169 (2000).

[5] A.G. Norman, H.L. Guthrey, M.R. Young, T. Song, T. Moriarty. Nat. Energy, 5, 326 (2020). DOI: $10.1038 / \mathrm{s} 41560-020-0598-5$

[6] [link] CPV Solar Cells. Last accessed: 24.12.2020. http://www.azurspace.com/index.php/en/products/productscpv/cpv-solar-cells.

[7] G. Siefer, A.W. Bett, K. Emery. In: Presented at the 19th European Photovoltaic Solar Energy Conference, Paris, (2004).

[8] V.D. Rumyantsev. Optics Express, 18 (S1), A17 (2010).

[9] [link] CPVMod - CPV Module in Modular Design. Last accessed: 24.12.2020.

https://www.ise.fraunhofer.de/en/research-projects/cpvmod

[10] E. Gerster, T. Gerstmaier, A. Gombert, R. Krause, S. Riesena, S. Wanka, T. Zech. AIP Conf. Proc., 1679, 040006 (2015). DOI: $10.1063 / 1.4931517$

[11] Е.А. Ионова, М.В. Уланов, Н.Ю. Давидюк, Н.А. Садчиков. ЖТФ, 86 (12), 87 (2016). [Е.A. Ionova, M.V. Ulanov, N.Yu. Davidyuk, N.A. Sadchikov, Tech. Phys. 61 (12), 1844 (2016).]

[12] V.A. Grilikhes, V.M. Andreev, A.A. Soluyanov, E.V. Vlasova, M.Z. Shvarts. Proc. of the Fourth Int. Conf. on Solar Concentrators for the Generation of Electricity or Hydrogen, El Escorial, Spain, 49, (2007).

[13] V.D. Rumyantsev, V.R. Larionov, P.V. Pokrovskiy. In: AIP Conference Proceedings, 1679, 050009 (2015). 